THE ACTIVE SUN 


\title{
SPECTRAL OBSERVATIONS OF SOLAR ACTIVITY AT METRE WAVE-LENGTHS
}

\author{
J. P. WILD \\ Radiophysics Laboratory, Sydney, Australia
}

This paper summarizes some recent results obtained with the solar radiospectroscope at Dapto (Sydney), which has been in operation since I952. The basic instrument is a receiver which sweeps through the frequency range $4^{0-240} \mathrm{Mc}$./s. every half a second. Each spectrum is displayed as an intensity-modulated line, and successive spectra are recorded photographically on continuously moving film to give a dynamic record of the spectrum.

In describing these results reference will be made to the three distinctive types of burst previously recognized. These are depicted in the idealized sketches of Fig. I, all being shown on the same time-scale.

\section{DERIVED VELOCITIES OF GORPUSGULAR STREAMS FROM THE SUN}

We have previously described the characteristic frequency drift in bursts of spectral types II and III. The drift is believed to be due to ionized matter streaming up through the solar atmosphere, exciting plasma oscillations at both fundamental and second-harmonic frequencies; as the matter passes into successively more rarefied regions of the corona, so these frequencies gradually decrease. Thus the frequency scales in Fig. I have been shown also as a scale of height above the solar photosphere. This scale is based on electron densities of the undisturbed corona given by Allen and van de Hulst. The scale is intended to refer only to radiation at the fundamental frequency of emission, and is not applicable to the second harmonic.

Velocities derived from the frequency drift of a number of bursts of types II and III are given in the upper histogram of Fig. 2. (In some cases type III drifts 'stop' before reaching the $40 \mathrm{Mc}$./s. level; these have been excluded from the histogram.) It is seen that the velocities lie in the range 300 to $700 \mathrm{~km}$. $/ \mathrm{sec}$. for type II bursts, and $2 \times 10^{4}$ to $2 \times 10^{5} \mathrm{~km}$. $/ \mathrm{sec}$. for type III bursts. 
Most type II bursts and certain type iI bursts occur at the time of solar flares, and the suggestion has been made that the two classes of stream correspond respectively to the geomagnetic storm particles which arrive at the earth $\mathrm{I}$ to 3 days after large flares, and to the cosmic rays which arrive about I hr. after large flares. The lower histograms of Fig. 2 show tentative
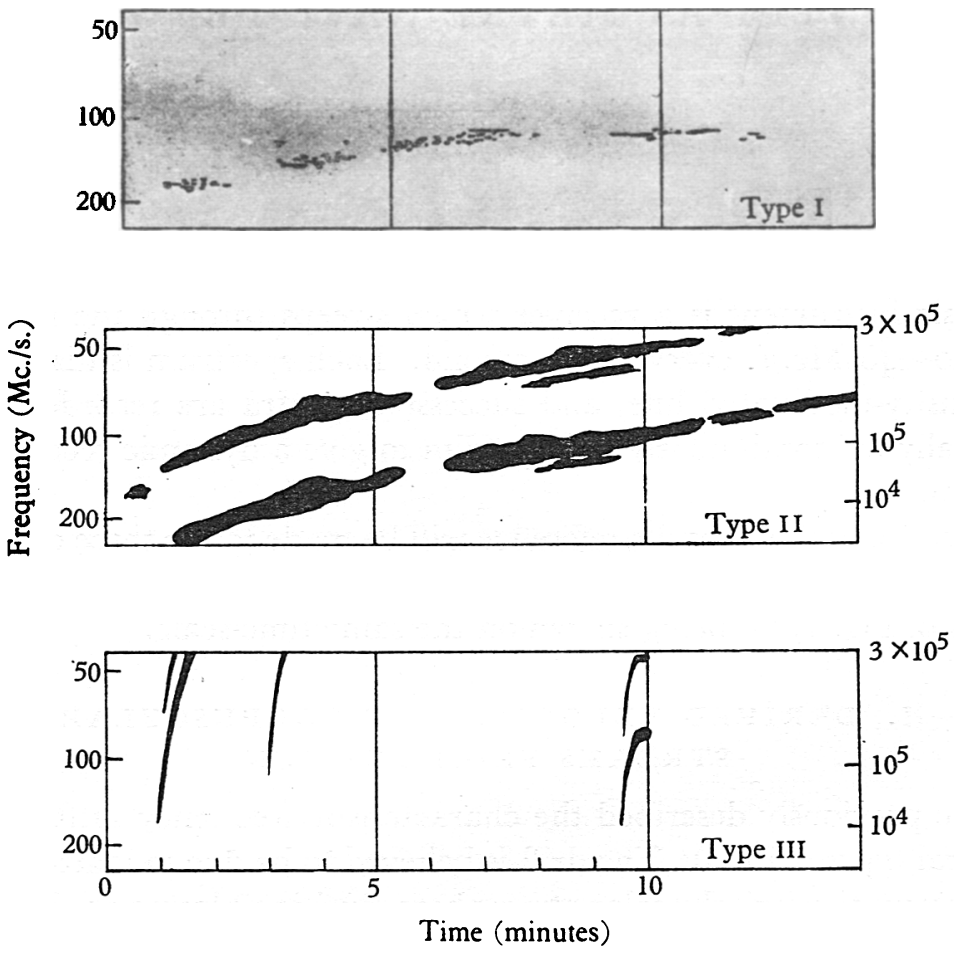

Fig. 1. Sketches showing idealized examples of the dynamic spectra of three distinctive types of solar radio disturbance. The scale given on the right-hand side gives the approximate height of the plasma-frequency level in the solar atmosphere. For types II and III the second harmonic is radiated as well as the fundamental; the height-scale refers to the latter only.

data on the velocities of these solar-terrestrial effects, inferred from published sun-earth time delays after flares.

The histogram indicates that any earth-bound corpuscles ejected from a flare with type III velocities would reach the earth at about the time when the cosmic-ray increase begins. On the other hand the energy of corpuscles with type III velocities ( $\sim 100 \mathrm{MeV}$ if the corpuscles are protons) is considerably less than the cosmic-ray energies. The possible association between the two phenomena is therefore obscure, and detailed simulta- 
neous observations of cosmic-ray intensities and type III bursts will be required to discover whether any connexion exists.

The type II velocities are distinctly slower than the velocities of known geomagnetic streams. However, it is important to note that the latter velocities apply only to the 'great' flares of importance $3^{+}$, while the limited sample of type II bursts so far observed correspond to smaller

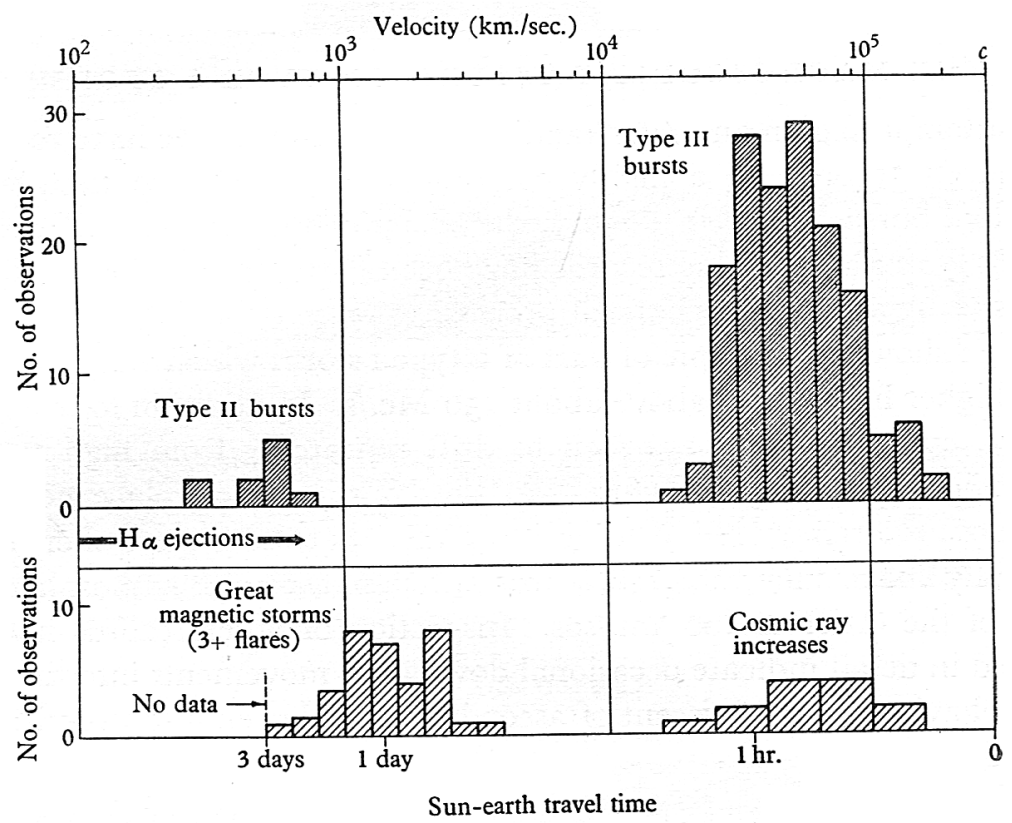

Fig. 2. Histogram (above) of velocities derived from the frequency drift of bursts of spectral type II and III. These velocities are compared (below) with those inferred from the sun-earth time delays of terrestrial magnetic storms and cosmic-ray increases, and with $\mathrm{H}_{\alpha}$ ejections from flares. The magnetic-storm histogram is that published by $\mathrm{H}$. W. Newton and W. Jackson, and the cosmic-ray data were collected by E. P. George (private communication).

flares. The type II velocities also correspond to the fastest observed $\mathrm{H}_{\alpha}$ ejections from flares. Hence the type in streams may correspond either to the geomagnetic particles or $\mathrm{H}_{\alpha}$ ejections (the latter two phenomena may themselves be related).

\section{TWO GLASSES OF SOLAR RADIO STORM}

We now recognize two distinct classes of solar radio storm (i.e. prolonged periods of disturbed radio emission lasting hours or days). One kind consists of a continuous sequence of the polarized, narrow-band type I 
bursts. These are often clustered within restricted but variable frequency bands and superposed on a slowly changing background continuum (Fig. $3 c$ ). The other consists of an intermittent sequence of the broad-band type III bursts occurring irregularly at a rate of the order of Io-1oo per hour (Fig. $3^{b}$ ). Both kinds of storm appear to be linked with sunspot activity. One kind can occur without the other, and both can occur together.

\section{THE MAGROSGOPIG PATTERN OF TYPE I STORMS}

In an attempt to gain an understanding of type I storms, we have recently reproduced records on a highly compressed time-scale, so that, while individual bursts lose their identity, the 'macroscopic' features of a storm are clearly revealed. These records have been derived from normal-speed records using a photo-mechanical process.

Fig. $3 d$ shows an example of part of a type I storm which was restricted to the higher frequencies above about $150 \mathrm{Mc}$./s. In addition to irregular features, emission bands are seen to drift repeatedly from high to low frequencies. Assuming as before that the emission takes place near the plasma or zero-refractive-index level, we infer that the sources of emission repeatedly make movements upwards through the solar atmosphere at speeds of the order of $100 \mathrm{~km}$. $/ \mathrm{sec}$. Inspection of other records not yet analyzed in detail indicate occasional downward movements into the sun. Such behaviour is reminiscent of ascending and descending prominence material.

\section{GOMBINED OBSERVATIONS OF SPEGTRUM AND POLARIZATION}

A simple technique has been devised by which the polarization of the received waves is determined at a number of frequencies across the spectrum. Two mutually perpendicular aerials are connected to the sweptfrequency receiver by unequal lengths of cable. When polarized radiation is incident upon the aerial system, the signals coming from the two elements interfere, causing the true spectrum to be modulated sinusoidally. The frequency interval between adjacent maxima of this modulation is determined by the difference in the two cable lengths, and the phase of the modulation by the type of polarization (left-hand or right-hand circular, linear, elliptical, etc.). The results largely confirm what had previously been inferred-that type I activity (bursts and background continuum) tend to be strongly polarized, while type II and type III bursts tend to be 

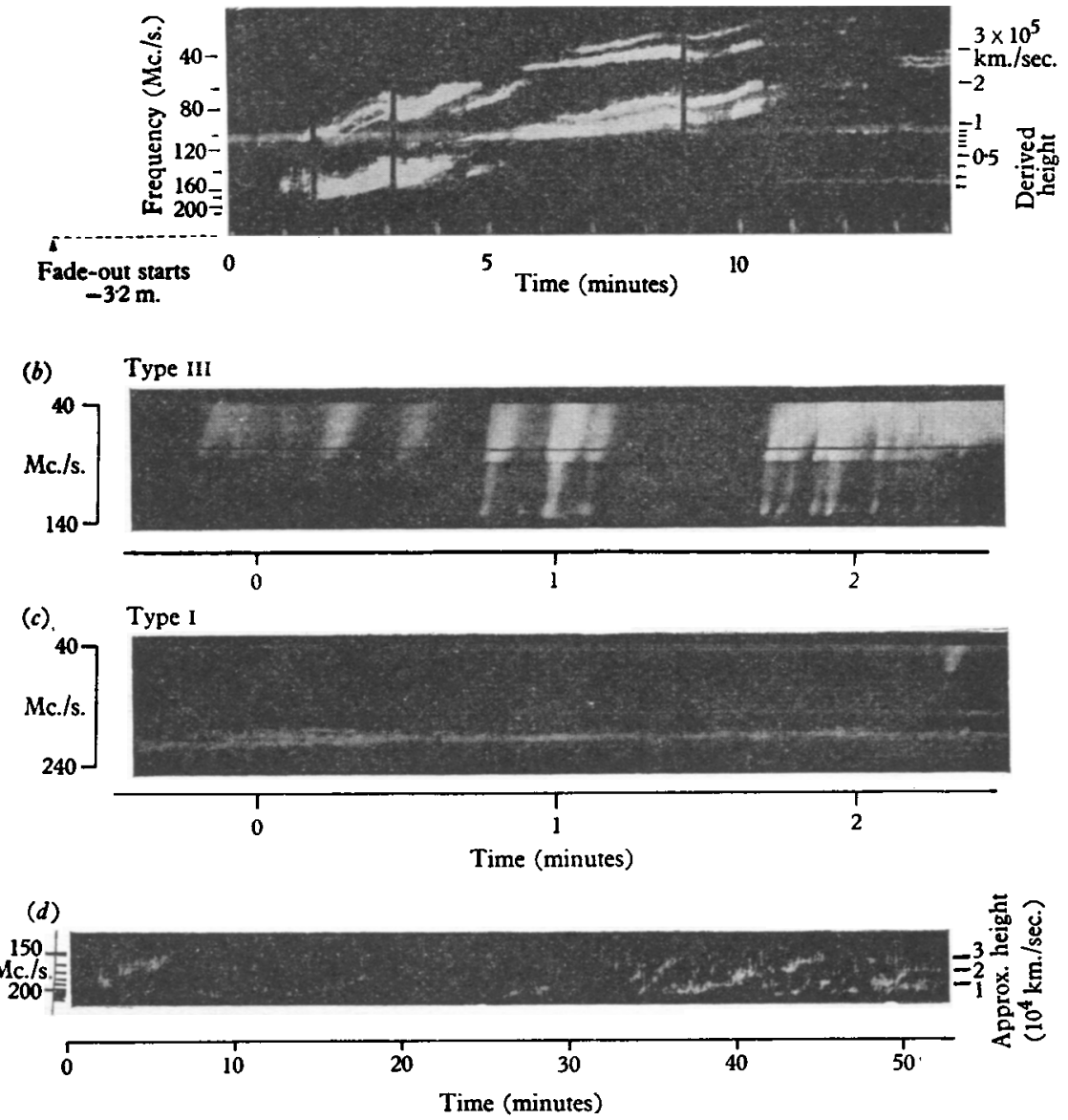

Fig. 3. Dynamic spectrograms of solar activity: (a) Type II burst accompanying a solar flare, showing two harmonic bands with internal fine structure-21 November 1952, 23.49 U.T. The horizontal streaks (e.g. at $120 \mathrm{Mc}$./s.) are instrumental in origin. (b) An intense part of a prolonged storm of type III bursts (in this case the intensification occurred at the time of a flare)4 October 1952, or. 58 U.T. (c) Part of a prolonged storm of type I bursts (an isolated type III burst is present near the right-hand edge) - 27 April 1953. (d) One hour's record of the same type I storm as in (c), shown on a highly compressed scale of time. Systematic frequency drifts are evident in this case.

\section{Note on conversion of time scales}

Figs. $3^{b}$ and $3^{c}$ are direct prints from the original records. In Figs. $3 a$ and $3 d$, however, the time scale of the original record has been compressed by a large factor. This was achieved by projecting the record on an opaque screen in which was cut a narrow slit parallel to and extending across the projected frequency scale. The slit was covered by a ground-glass screen capable of being photographed with a recording camera placed behind the screen. In operation the original record was passed continuously through the projector while fresh film was passed through the recording camera at a slower speed determined by the degree of compression required. 
unpolarized. However, some type III bursts observed early in the morning and late in the evening were found to be polarized, suggesting a terrestrial influence in these cases.

\section{BIBLIOGRAPHY}

Wild, J. P. and McCready, L. L. Aust. J. Sci. Res. A, 3, 387, 1950.

Wild, J. P., Murray, J. D. and Rowe, W. C. Aust. J. Phys. 7, 439, 1954.

Wild, J. P., Roberts, J. A. and Murray, J. D. Nature, 173, 532, 1954. 